\title{
UNSTEADY AERODYNAMIC EFFECTS ON THE FLIGHT CHARACTERISTICS OF AN F-16XL CONFIGURATION
}

\author{
Zhongjun Wang and C. Edward Lan' \\ Department of Acrospace Engincering \\ University of Kansas \\ Lawrence, KS 66045 \\ and \\ Jay M. Brandon" \\ Vehicle Dynamics Branch \\ NASA Langley Research Center \\ Hampton, VA
}

\begin{abstract}
$I_{x y}, I_{x z}, I_{y z} \quad$ product of inertia

Unsteady aerodynamic models based on windtunnel $F_{\text {laco, }}, F_{m \text { ecro }}, F_{n \text { ecro, }}, T_{1}, T_{m}, T_{n}$ aerodynamic and thrust moments about forced oscillation test data and analyzed with a fuzzy logic algorithm are incorporated into an F-16XL flight simulation code. The reduced frequency needed in the unsteady models is numerically calculated by using a limited prior time history of state variables in a least-square sense. Numerical examples are presented to show the accuracy of the calculated reduced frequency. Oscillatory control inputs are employed to demonstrate the differences in the flight characteristics based on unsteady and quasi-steady aerodynamic models. Application of the unsteady aerodynamic models is also presented and the results are compared with one set of F16XL longitudinal maneuver flight data. It is shown that the main differences in dynamic response are in the lateraldirectional characteristics, with the quasi-steady model being more stable than the flight vehicle, while the unsteady model being more unstable. Similar conclusions can also be made in a simulated rapid sideslipping roll. To improve unsteady aerodynamic modeling, it is recommended to acquire test data with coupled motions in pitch, roll and yaw.

\section{Nomenclature}

w

$\mathrm{p}, \mathrm{q}, \mathrm{r}$

$\mathrm{u}, \mathrm{v}, \mathrm{w}$

$\mathrm{V} \quad$ total velocity

$F_{x \text { zaco, }}, F_{y \text { zeco }}, F_{z \text { acro, }} T_{x}, T_{y}, T_{z}$ aerodynamic forces and thrust along the $\mathrm{x}-, \mathrm{y}$ - and $\mathrm{z}$-axes, respectively

$\alpha \quad$ angle of attack

$\beta \quad$ angle of sideslip

$\delta_{e}, \delta_{a}, \delta_{r} \quad$ control deflections in pitch, roll and yaw, respectively

$\delta_{\mathrm{T}} \quad$ throttle position

$\phi, \theta, \psi \quad$ Euler angles in roll, pitch, and yaw,

respectively

k reduced frequency

b span length

$\bar{c}$

$\mathrm{C}_{\mathrm{ij}} \quad$ directional cosines

Introduction

Aircraft dynamic characteristics have mostly been

\footnotetext{
-Graduate Research Assistant

+ J. L. Constant Distinguished Professor. Associate Fellow AlAA

"Acrospace Engincer, Senior Member AIAA

Copyright ( 2000 by the Amcrican Institutc of Acronautics 1 and Astronautics. Inc. All rights reserved.
} 
studicd with a quasi-stcady acrodynamic model in the past In a quasi-stcady model, the dynamic effects on aerodynamic coefficients are assumed to be lincarly proportional to the angular rates and time rates of change of variables with the coefficients being typically measured at a fixed frequency. That is, the lag effect exhibited by the varying reduced frequency along a mancuvering fight path, and the corresponding dynamic stall effect are not properly accounted for. The present investigation on the unsteady aerodynamic effect is undertaken in view of the fact that the lag and dynamic stall effects may play an important role in aircraft stability characteristics and performance ${ }^{1,2}$ in some flight conditions.

To determine the unsteady aerodynamic effects, harmonic forced oscillation tests were conducted., ${ }^{3}$ These test data were then analyzed to establish the aerodynamic models for use in the flight simulation. In the process of application, the most uncertain quantity to be determined is the reduced frequency. It is straightforward to calculate the reduced frequency of harmonic oscillation motion used in the test. However, the actual aircraft motion cannot be described by one single harmonic oscillation, and the reduced frequency of the actual motion will not be constant. One way to determine the reduced frequency of aircraft motion is an analytical method which assumes the mean angle of attack and the amplitude being the same as those used in the harmonic oscillation tests. ${ }^{1}$ If the forced oscillation tests were performed at a constant mean angle of attack and amplitude, the analytical method should produce reasonable values of the "equivalent" reduced frequency. On the other hand, when the wind tunnel data are obtained at different mean angles of attack and amplitudes, this analytical method would not be applicable. One way to determine the equivalent reduced frequency is a numerical one.

In the present paper, we will demonstrate one numerical method to determine the reduced frequency for use in flight simulation. The method is then employed, together with the fuzzy-logic aerodynamic models established previously ${ }^{3,4}$, in an F-16XL simulation code to investigate the unsteady aerodynamic effects on flight dynamics.

\section{Aircraft Dynamic Model}

The flight simulation is implemented by the following 6-degrec-of-frecdom model:

$$
\begin{aligned}
& \dot{u}=r-q w^{\prime}+a_{x, b} \\
& \dot{v}=p w-r u+a_{y, b} \\
& \dot{w}=q u-p v+a_{z, b} \\
& \dot{p}=\dot{H}_{x} A_{11}+\dot{H}_{y} A_{12}+\dot{H}_{z} A_{13} \\
& \dot{q}=\dot{H}_{x} A_{21}+\dot{H}_{y} A_{22}+\dot{H}_{z} A_{23} \\
& \dot{r}=\dot{H}_{x} A_{31}+\dot{H}_{y} A_{32}+\dot{H}_{z} A_{33}
\end{aligned}
$$

where

$$
\begin{gathered}
a_{x, b}=g\left(a_{x}+C_{13}\right) \\
a_{y, b}=g\left(a_{y}+C_{23}\right) \\
a_{z, b}=g\left(-a_{n}+C_{33}\right) \\
a_{x}=\left(T_{x}+F_{x, \text { aero }}\right) / W \\
a_{y}=\left(T_{y}+F_{y, \text { aero }}\right) / W \\
a_{n}=-\left(T_{z}+F_{z, \text { arro }}\right) / W
\end{gathered}
$$

$$
\begin{aligned}
& H_{x}=p I_{x}-q I_{x y}-r I_{x x} \\
& H_{y}=-p I_{x y}+q I_{y}-r I_{y z} \\
& H_{z}=-p I_{x z}-q I_{y z}+r I_{z} \\
& \dot{H}_{x}=r H_{y}-q H_{z}+F_{l, a r r o}+T_{l} \\
& \dot{H}_{y}=p H_{z}-r H_{x}+F_{m, a r r o}+T_{m} \\
& \dot{H}_{z}=r H_{x}-q H_{y}+F_{\text {nadro }}+T_{n}
\end{aligned}
$$

$$
\phi=\tan ^{-1}\left(C_{23} / C_{33}\right)
$$$$
\theta=-\sin ^{-1}\left(C_{13}\right)
$$$$
\psi=\tan ^{-1}\left(C_{12} / C_{11}\right)
$$$$
A_{11}=\left(I_{y} I_{z}-I_{y z}^{2}\right) / D
$$$$
A_{12}=A_{21}=\left(I_{y 2} I_{x 2}+I_{z x}\right) / D
$$$$
A_{22}=\left(I_{x} I_{2}-I_{x_{2}}^{2}\right) / D
$$$$
A_{23}=A_{32}=\left(I_{x y} I_{x z}+I_{z y z} I_{y z}\right) / D
$$$$
A_{33}=\left(I_{Y} I_{y}-I^{2}\right) / D
$$$$
A_{13}=A_{31}=\left(I_{x y} I_{y z}+I_{y x z} I_{x z}\right) / D
$$$$
D=I_{x y} I_{z}-I_{x} I_{y z}^{2}-I_{y} I_{z z}^{2}-I I_{x y}^{2}-2 I_{x y} I_{x z} I_{y z}
$$

The flight state variables $(u, v, w, p, q, r)$ are calculated by integrating these dynamic equations. The

following auxiliary cquations are then uscd to obtain the 
angle of attack and sideslip angle and their time rates :

$$
\begin{aligned}
& \alpha=\tan ^{-1}(w / u) \\
& \dot{\alpha}=(u \dot{w}-u \dot{u}) /\left(u^{2}+w^{2}\right) \\
& \beta=\sin ^{-1}(v / V) \\
& \dot{\beta}=(V \dot{v}-v \dot{V}) /\left(V^{2} \cos \beta\right) \\
& V=\sqrt{u^{2}+v^{2}+w^{2}}
\end{aligned}
$$

\section{Aerodynamic Models}

\section{Quasi-steady model}

For quasi-steady aerodynamics, the terms $\mathrm{F}_{\mathrm{x} \text { acro }}$,

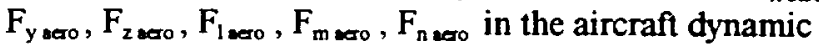
model are functions of flight state variables and control surface deflection angles, i.e.,

$$
F_{x, y, z, l, m, n, \text { aero }}=h(\alpha, \beta, p, q, r, V, H, \delta)
$$

where $\delta$ stands for the control deflection angle. When the flight state is known, it is straightforward to obtain the corresponding aerodynamic forces and moments through interpolation. The aerodynamic effects caused by the time rate of angle of attack are not included in the present quasisteady aerodynamic model for the F-16XL.

\section{Unsteady aerodynamic model}

The longitudinal and lateral-directional unsteady aerodynamic models were established based on the results of large-amplitude pitch, roll and yaw forced oscillation tests, and a limited amount of rotary balance data. ${ }^{3,4}$ The tests were conducted in separate pitch, roll and yaw motions. These models were set up to have the following functional forms:

Longitudinal unsteady aerodynamics model:

$$
C_{L}, D, m, n, \ell_{,} y=f\left(\alpha, \dot{\alpha}, \ddot{\alpha}, k_{1}, \beta, \delta_{e}\right)
$$

Lateral-directional unsteady aerodynamics model:

$$
C L, D, m, n, \ell, v=g\left(\alpha, \phi, \dot{\phi}, k_{2}, \psi, \dot{\psi}\right)
$$

where the subscripts $L, D, m, n, l, y$ stand for lift. drag. pitching. yawing and rolling moments, and side force, respeclively. The reduced frequency $k_{\text {, }}$ is computed from $\alpha(1)$ and $q(1)$, representing the longitudinal unsteady motion characteristics. On the other hand, the reduced frequency $k_{2}$ is calculated from the lateral-directional unsteady motion, mainly from the rolling motion if it is present. Note that there are no unsteady aerodynamic data available with the lateral-directional control surfaces deflected. Only the elcvator effect is included in the longitudinal model.

In flight simulation, the motion variables $\alpha, \phi, \psi$ and their time rates are calculated at every time instant. The calculation of reduced frequency $k_{1}$ is discussed in the next section. For the lateral-directional case, two reduced frequencies can be obtained separately from $\phi, \dot{\phi}$, and $\psi$, $\dot{\psi}$. In most cases, $\mathrm{k}_{2}$ will be calculated from $\phi$ and $\dot{\phi}$ unless there is no aileron input. In this latter case, $\psi$ and $\dot{\psi}$ will be used to obtain $\mathrm{k}_{2}$. This approach is based on the fact that the lateral-directional motion variables tend to have similar time histories.

In addition to the aforementioned unsteady aerodynamic models, the existing static aerodynamic model in the simulation code is used to provide the basic aerodynamic properties. The unsteady aerodynamic effects are added to the basic static aerodynamics according to the following expressions:

Longitudinal unsteady aerodynamic increments:

$$
\begin{aligned}
& \Delta C_{\text {unsteadyl }}=\Delta C_{L, D, m, n, \ell, y}=f\left(\alpha, \dot{\alpha}, \ddot{\alpha}, k_{1}, \beta, \delta_{e}\right) \\
& -f\left(\alpha, \dot{\alpha}=0, \ddot{\alpha}=0, k_{1}=0, \beta, \delta_{e}\right)
\end{aligned}
$$

Lateral-directional unsteady aerodynamic increments:

$$
\begin{aligned}
& \Delta C_{\text {unsteady } 2}=\Delta C L, D, m, n, \ell, y=g\left(\alpha, \phi, \dot{\phi}, k_{2}, \psi, \dot{\psi}\right) \\
& -g\left(\alpha, \phi, \dot{\phi}=0, k_{2}=0, \psi, \dot{\psi}=0\right)
\end{aligned}
$$

Generally, $\dot{\phi}$ and $\dot{\psi}$ are replaced by $\mathrm{p}$ and $\mathrm{r}$, respectively. Therefore, the total aerodynamic coefficients at every time instant are expressed by:

$$
C_{L, D, m, n, \ell, y}=C_{\text {static }}+\Delta C_{\text {unsteady } 1}+\Delta C_{\text {unsteady } 2}
$$

For the purpose of stability analysis, the derivatives of aerodynamic coefficients with respect to the angle of attack etc. can also be derived from the above equations. In addition to the numerical central difference method and spline interpolation to obtain these derivatives, the small-amplitude harmonic oscillatory methods will be used and compared 


\section{Equivalent Reduced Frequency}

In the present unsteady acrodynamic models, different types of forced oscillation test results (different mean angles of attack and different amplitudes) are used as source data to train the fuzzy logic models. This was done in the testing to accommodate a wider range of reduced frequency without exceeding the loading capability of the measuring balance. As stated previously, the angle of attack

$\alpha$, and the time rate of angle of attack $\alpha$ are part of the

input parameters to calculate the unsteady aerodynamic coefficients in the longitudinal fuzzy logic model. The time history of these variables is fitted with one of a harmonic motion at that instant, i.e.,

$$
\begin{aligned}
& \alpha(t)=\bar{\alpha}+a \cos (\omega t+\bar{\phi}) \\
& \dot{\alpha}(t)=-a \omega \sin (\omega t+\bar{\phi})
\end{aligned}
$$

where those terms on the left hand side are given and the unknowns are $\bar{\alpha}$, the local mean angle of attack, " $a$ ", the local amplitude of the harmonic motion, $\bar{\phi}$, the phase lag, and $\omega$, the angular frequency. The reduced frequency $k_{1}$ and $k_{2}$ are defined as

$$
k_{1}=\omega \bar{c} / V, k_{2}=\omega b / 2 V
$$

The frequency $\omega$ and other parameters are calculated through an optimization method by minimizing the following cost function (least squares)

$$
\begin{aligned}
J= & \sum_{i=1}^{n}\left[\alpha_{i}-\left(\bar{\alpha}+a \cos \left(\omega t_{i}+\phi\right)\right)\right]^{2}+ \\
& \sum_{i=1}^{n}\left[\alpha_{i}-\left(-a \omega \sin \left(\omega t_{i}+\phi\right)\right)\right]^{2}
\end{aligned}
$$

where $\mathbf{n}$ is the number of data points used, and is assumed to be 20 (equivalent to $0.7 \mathrm{sec}$ ) in the present application. Those twenty points preceding the current time are employed in the above equation. The least-square method is found to converge well and gives reasonably accurate results. The lateral-directional equivalent reduced frequency is computed in the same manner.

\section{Numerical Results and Discussion}

Equivalent Reduced Frequency and Pitch Damping
Assume the number of points in the motion history preceding the current instant is $n$. If $n>20$. it is set to 20 However, if $\mathbf{n}<20$, then only those available points are used to determine the unknowns. As is well known, the initial values assumed will significantly affect the convergence of numerical optimization. Therefore, the initial values of the unknowns for the current time segment are always taken to be the results from the previous segment. At the beginning, the initial values of mean $\alpha$ $(\bar{\alpha})$ and amplitude $(a)$ are assumed to be $35.0 \mathrm{deg}$. (the value used in the windtunnel testing), and the difference between the actual $\alpha$ and the assumed mean $\alpha$, respectively. The initial values of angular frequency and phase angle are 1.0 and $\pi$, respectively.

Figure 1 presents the comparison between the assumed harmonic motion with $\omega=\pi / 2$ and the predicted results. Except a small deviation when $n<20$, the present method is shown to predict accurate results. The method has also been tested for various other assumed harmonic motions with similar good results (not shown).

The convergence of the present algorithm is fast. For an $\alpha(t)$ response with 150 points, it took only one second of the CPU time to calculate the reduced frequency at all 150 points on an Alpha VAX machine.

The variation of longitudinal dynamic derivatives with the angle of attack based on the concept of small amplitude harmonic oscillation is presented in Figure 2. These dynamic derivatives are calculated by integrating the out-of-phase aerodynamic coefficients obtained from the fuzzy logic model. The amplitude is assumed to be 5 degrees and two different reduced frequencies are presented in the calculation. The pitching moment is referenced to a pitch center at $0.558 \bar{c}$. The results show that predicted pitch damping derivatives are mostly positive because of the aft pitch center location. Their sign agrees with the hysteresis direction in that a clockwise loop produces a positive value for the damping derivatives. Their magnitude is decreased with increasing reduced frequency, having the same trend of variation with that calculated by the indicial integration method. ${ }^{5}$ This exercise demonstrates the generality and usefulness of fuzzy logic models as steady and unstcady acrodynamic data base

Dynamic Response to Oscillatory Inpu!

The aerodynamic moment reference point of the 
F-16XL simulation code is at $\mathrm{x}_{\mathrm{sum} 2}=0.45 \bar{c}$, being different from the ones used in the longitudinal and lateraldirectional wind tunnel tests. Therefore, the pitching moment and yawing moment cocficients obtained from the fuzzy logic models must first be transferred before being incorporated into the simulation code by using the following expressions:

$$
\begin{aligned}
& C_{m}=C_{m \text { model }}+C_{L}\left(x_{\text {stim } 2}-x_{\text {long }}\right) \\
& C_{n}=C_{n \text { model }}+C_{y}\left(x_{\text {sim } 2}-x_{\text {lat }}\right)
\end{aligned}
$$

Note that $\mathrm{x}_{\text {long }}=0.558 \bar{c}$ and $\mathrm{x}_{\text {bat }}=0.46 \bar{c}$.

To see the differences in the dynamic response with unsteady and quasi-steady aerodynamic models, the following oscillatory elevator input by the pilot is assumed:

$$
\delta_{\text {epiot }}=15.0+25.0 \sin (2 \pi / 8 \mathrm{t}) \text {, in deg. }
$$

The resulting dynamic response is presented in Figure 3. The lateral-directional state variables, such as the sideslip angle, roll rate and yaw rate etc., are zero. Two methods in calculating the dynamic derivatives with the unsteady aerodynamic model are presented: one with the small amplitude oscillation method mentioned earlier and the other based on a central difference method. For the latter, the following equation is used:

$$
\begin{aligned}
& C_{L g}=\left[f\left(\alpha, \Delta, \ddot{\alpha}, k_{1}, \beta, \delta_{e}\right)-f\left(\alpha,-\Delta, \ddot{\alpha}, k_{1}, \beta, \delta_{e}\right)\right] /(2 \bar{\Delta})(13) \\
& \bar{\Delta}=\bar{c} \Delta /(2 V)
\end{aligned}
$$

where $\Delta=\mathrm{q}+\dot{\alpha}$ if the sum is greater than $5 \mathrm{deg} . / \mathrm{sec}$.; otherwise, $\Delta=5 \mathrm{deg} . / \mathrm{sec}$. For the lateral-directional derivatives, the following expression is used:

$C_{\ell p}=\left[g\left(\alpha, \phi, p+\Delta p, k_{2}, \psi, r\right)-g\left(\alpha, \phi, p-\Delta p, k_{2}, \psi, r\right)\right] /(2 \Delta \bar{p})(14)$

where $\Delta p=3$ deg./sec. and $\Delta \bar{p}=b \Delta p /(2 V)$. The yaw damping derivative is calculated in a similar manner. Note that pitching derivatives are calculated differently to avoid the effect of static data when the dynamic data are not symmetrical about the static data. The calculated results indicate that the derivatives by the small amplitude method vary in a more orderly manner as compared with the central difference method: but the order of magnitude is comparable. One reason for this is that in the small amplitude method. the results depend only on $\alpha$ and $\mathrm{k}$ : while the central difference method depends on $\alpha$ as well in addition to $\alpha$ and $\mathrm{k}$. Onc important difference between the unstcady model and the quasi-stcady model is that the latter features less pitch damping at low $\alpha$ and higher damping (i.c., values being more negative) at high $\alpha$, being opposite to what the unsteady model indicates. The lift duc to pitching is in general much smaller with the quasi-steady model. Note that during the simulation, the existing control system is still operational. The resulting angle of attack time history is seen to be the same for both the quasi-steady and unsteady models. This is achieved by the control system with different final elevator deflection angles to compensate for the differences in dynamic characteristics. Later, a test case will be shown that this is not the case in other unsteady flight conditions where the control system designed with the quasi-steady data becomes inadequate. In the following, all stability derivatives will be calculated with the central difference method.

\section{Comparison with Flight Test Data}

The longitudinal dynamic response is first calculated with the quasi-steady aerodynamic model, the pilot longitudinal control $\left(\delta_{\text {cpiou }}\right)$ being the only input, in addition to the contributions from flight control systems. The net control deflections are plotted as $\delta_{e} \delta_{a}$, and $\delta_{r}$. The initial flight state variables are found to be the same as the initial flight data with a center of gravity location at $\mathrm{x}_{\mathrm{og}}=0.45923 \bar{c}$. Figure 4 shows the simulation results in comparison with flight data. Although the longitudinal responses $(\alpha, \theta$ and $q)$ follow the trend of flight data well, there are time periods in which the predicted $\alpha$ is much lower than the data. These are also the time periods with lower magnitude in pitch damping. The lateral-directional motions are not excited at all, although there are some initial inputs from the control system. The aforementioned longitudinal responses are repeated with the unsteady aerodynamic models as shown in Figure 5. Assuming that the control system in the simulation code models accurately the one in the flight vehicle, the discrepancy in the responses must be caused by the static aerodynamic data which are also used in the unsteady models. The biggest differences between these two aerodynamic models occur in the lateral-directional responses. However, the excited lateral-directional motions with the unsteady models appear to be more unstable than what the flight data show, in particular in roll and sideslip.

\section{$\underline{\text { Sideslipping Roll }}$}

To help cxplain the acrodynamic causes of some 
loss-of-control flight conditions, such as the USAir Flight 427 accident in $1994^{\circ}$, the initial flight scenarios with a hard-over rudder malfunction will be postulated with the present F-16XL model. The present configuration is trimmed at $M=0.1718$ and $6000 \mathrm{ft}$. of altitude. With the longitudinal stick fixed at the trim position, the lateraldirectional control systems are disconnected; but the longitudinal system is left on. In the initial 1.5 seconds, a left bank is created by applying aileron and at the same time the rudder is set at a nose-left $20 \mathrm{deg}$. After 1.5 seconds, right aileron is applied to try to make the wing level. These are the scenarios indicated in Ref. 6 . The results of simulation are presented in Figure 6 . It is seen that with the quasi-steady aerodynamic model, a positive sideslip up to 12 deg. can be quickly generated while the angle of attack is increased to $36 \mathrm{deg}$. by the control system to counteract perhaps the increasing negative pitch attitude. Because of the positive sideslip, the resulting rolling moment overpowers the right aileron and the configuration keeps rolling to the left. Therefore, rapid sideslipping roll may generate loss-of-control flight conditions.

If the unsteady aerodynamic models are used in the simulation, the situation gets worse, with the aircraft responses in $\alpha$ and $\beta$ being divergent within five seconds. Typically, unsteady aerodynamics will make an unstable or marginally stable configuration with quasi-steady aerodynamic models more unstable. In the present case, the main reasons for the divergence are $C_{m \alpha}$ being positive and $\mathrm{C}_{n \mathrm{p}}$ being negative as shown in Figure 7. Based on the present simulation, even maintaining a small rudder deflection will make the model highly unstable (not shown). Whether these highly unstable conditions with the unsteady aerodynamic models exist can only be verified with more appropriate test data. These needed data should be those obtained with coupled motions in pitch, roll and yaw. From a theoretical point of view, this is because the lateral-directional aerodynamics, such as roll and yaw damping, depend on wing boundary layer characteristics and the associated wake, which in turn strongly depend on the angle of attack and its time rate of change. Another implication is that these appropriate unsteady aerodynamic data may be needed in control system design.

\section{Concluding Remarks}

Longitudinal and lateral-directional unstcady aerodynamic models obtained in separate wind tunnel testing were incorporated into an F-16XL simulation code which was based on a conventional quasi-stcady acrodynamic model. The resulting code was employed to investigate the differences in flight characteristics with these two types of aerodynamic models. For the unsteady model, the reduced frequency needed in the model was calculated with a least-square method to fit a harmonic variation to prior time history of state variables over about 0.7 second. Methods to extract dynamic derivatives while performing time integration of dynamic equations were developed based on the concept of central differences and small-amplitude harmonic oscillation. These two methods showed similar results which were significantly different from the original quasi-steady data. Flight data in a longitudinal maneuver were compared with the simulation results. The main differences in dynamic response based on these two types of aerodynamic models were the lateraldirectional responses induced by the longitudinal motion. For the quasi-steady model, the lateral-directional motion was not excited, as compared with the flight data. On the other hand, the unsteady model produced a more unstable motion than what the flight data indicated. In a simulated sideslipping roll, again the configuration was shown to be more directionally unstable with an unsteady model. To improve the unsteady aerodynamic modeling, test data with coupled motions in pitch, roll and yaw would be needed.

\section{Acknowledgment}

This research was supported by NASA Grant NAG-1-1821 for the first two authors.

\section{References}

' Hu, C. C., Lan, C. E. and Brandon J. M. "Unsteady Aerodynamic Models for Maneuvering Aircraft", AIAA Paper 93-3626CP, August, 1993.

${ }^{2}$ Rotary-Balance Testing for Aircraft Dynamics, AGARD Advisory Report, No. 265, 1991

${ }^{3}$ Wang, Z., Lan, C.E. and Brandon, J. M."Fuzzy Logic Modeling of Nonlinear Unsteady Aerodynamics", AIAA 98-4351, August, 1998.

4 Wang, Z., Lan, C.E. and Brandon, J. M."Fuzzy Logic Modeling of Lateral-Directional Unsteady Aerodynamics". AIAA 99-4012, August, 1999.

${ }^{5}$ Lin, G., Songster, T. and Lan C. E. "Effect of High-alpha Unsteady Aerodynamics on Longitudinal Dynamics of an F-18 Configuration" AlAA 95-3488, August, 1995. "Dornheim, M. A. "737 Simulation Recreates Accident Scquence" Aviation Heek and Space Technologv. March 22,1999, p. 42. 

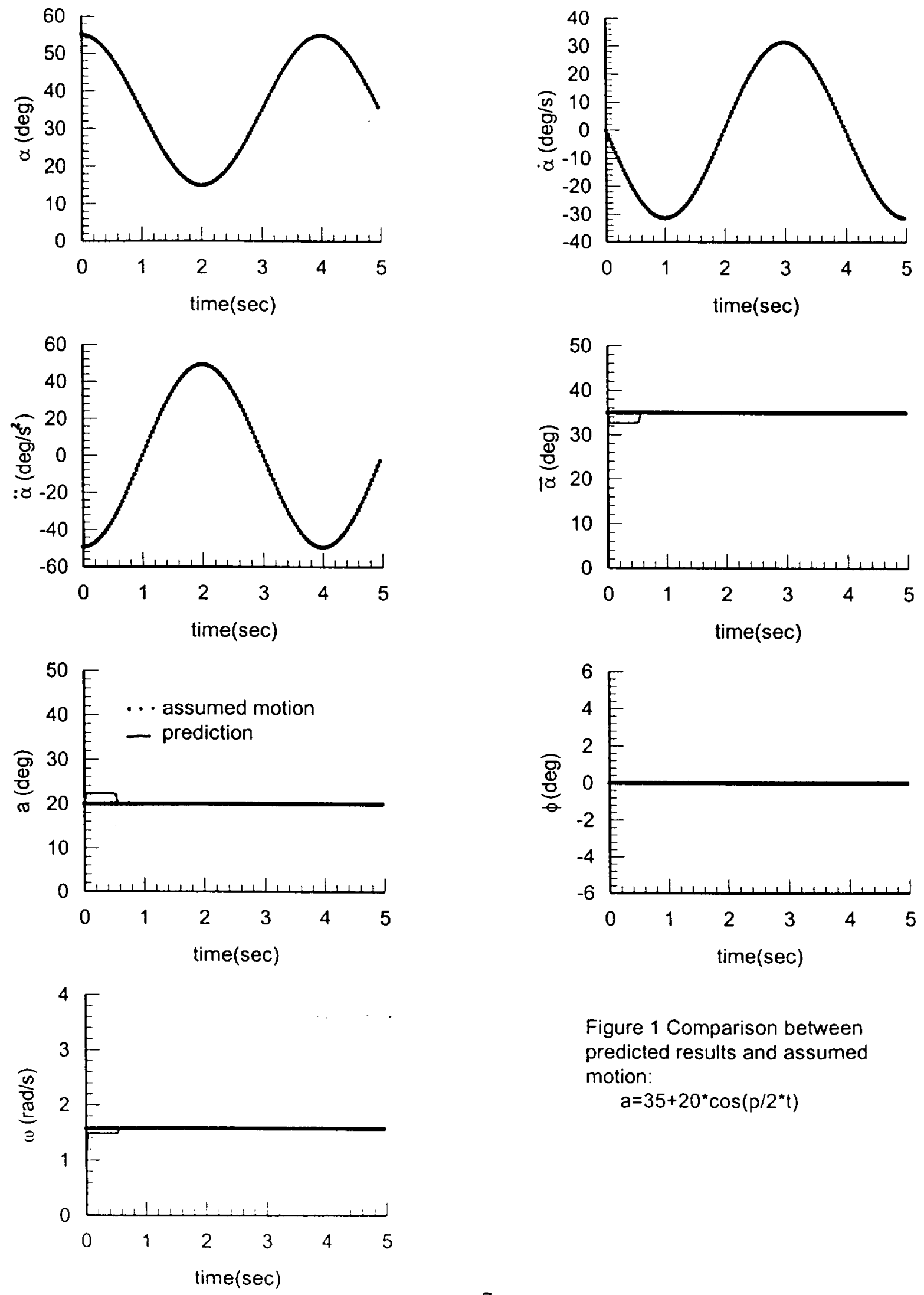

Figure 1 Comparison between predicted results and assumed motion:

$a=35+20^{\star} \cos \left(p / 2^{\star} t\right)$ 

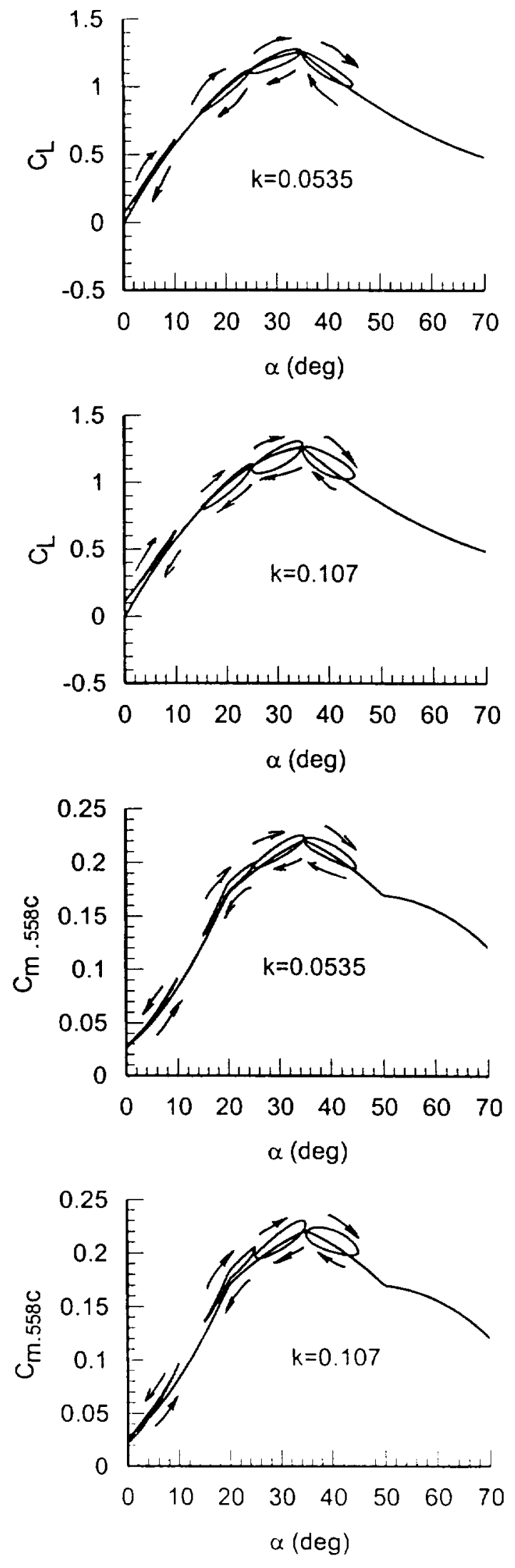
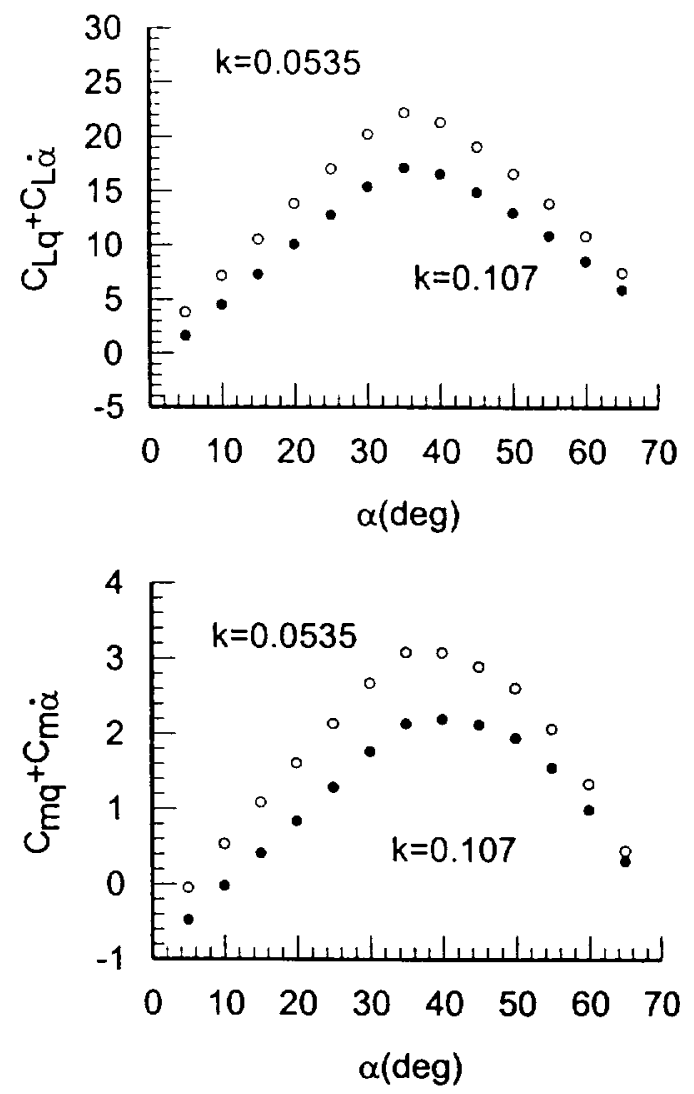

Figure 2 Static aerodynamics and calculated dynamic derivatives based on samll amplitude harmonic oscillation(amplitude: $a=5 \mathrm{deg}$ ) 

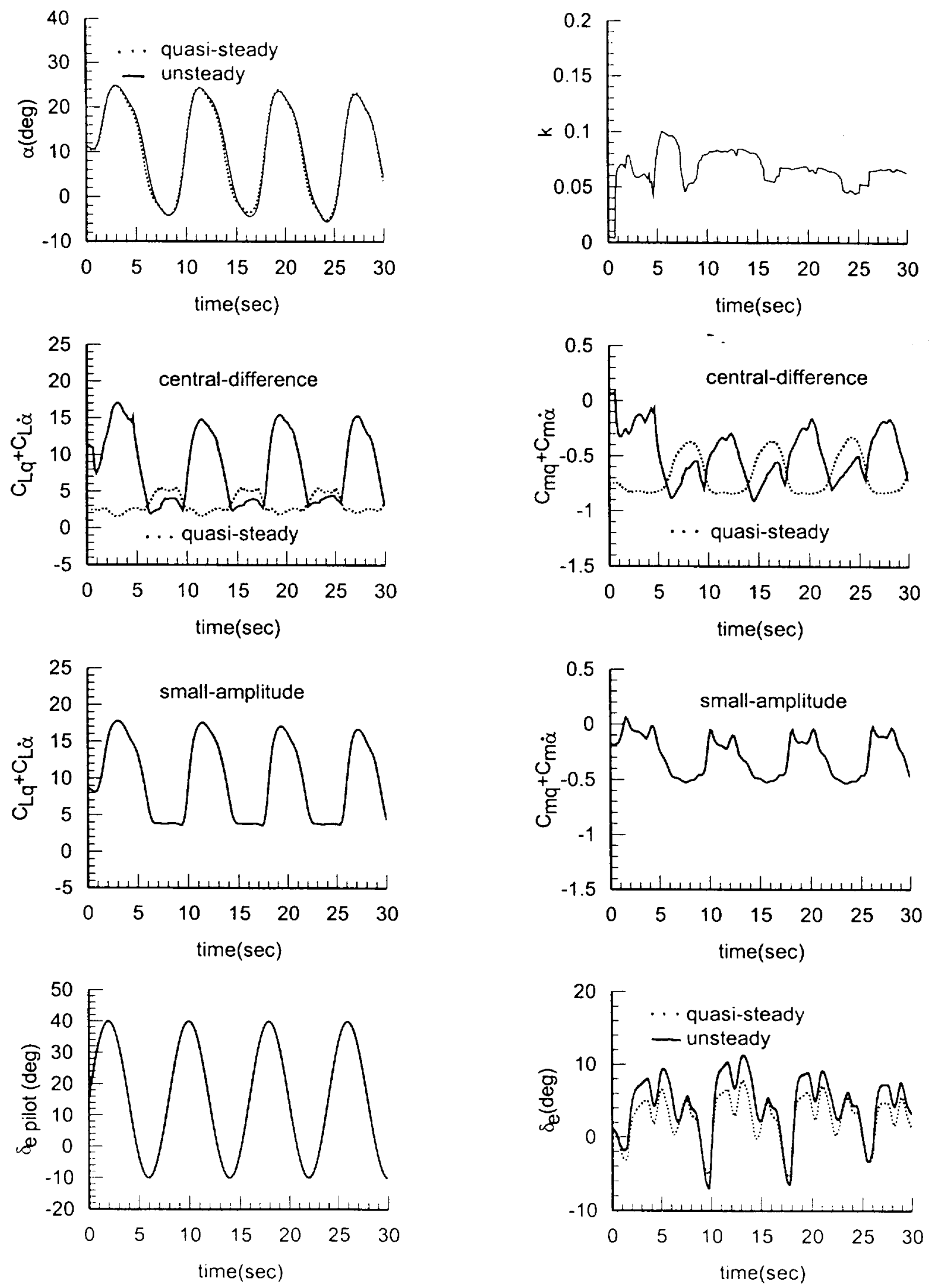

Figure 3 Simulation results with unsteady aerodynamic model and quasi-steady aerodynamic model(pilot elevator input only). $x c g=0.45923 \bar{c}$ 

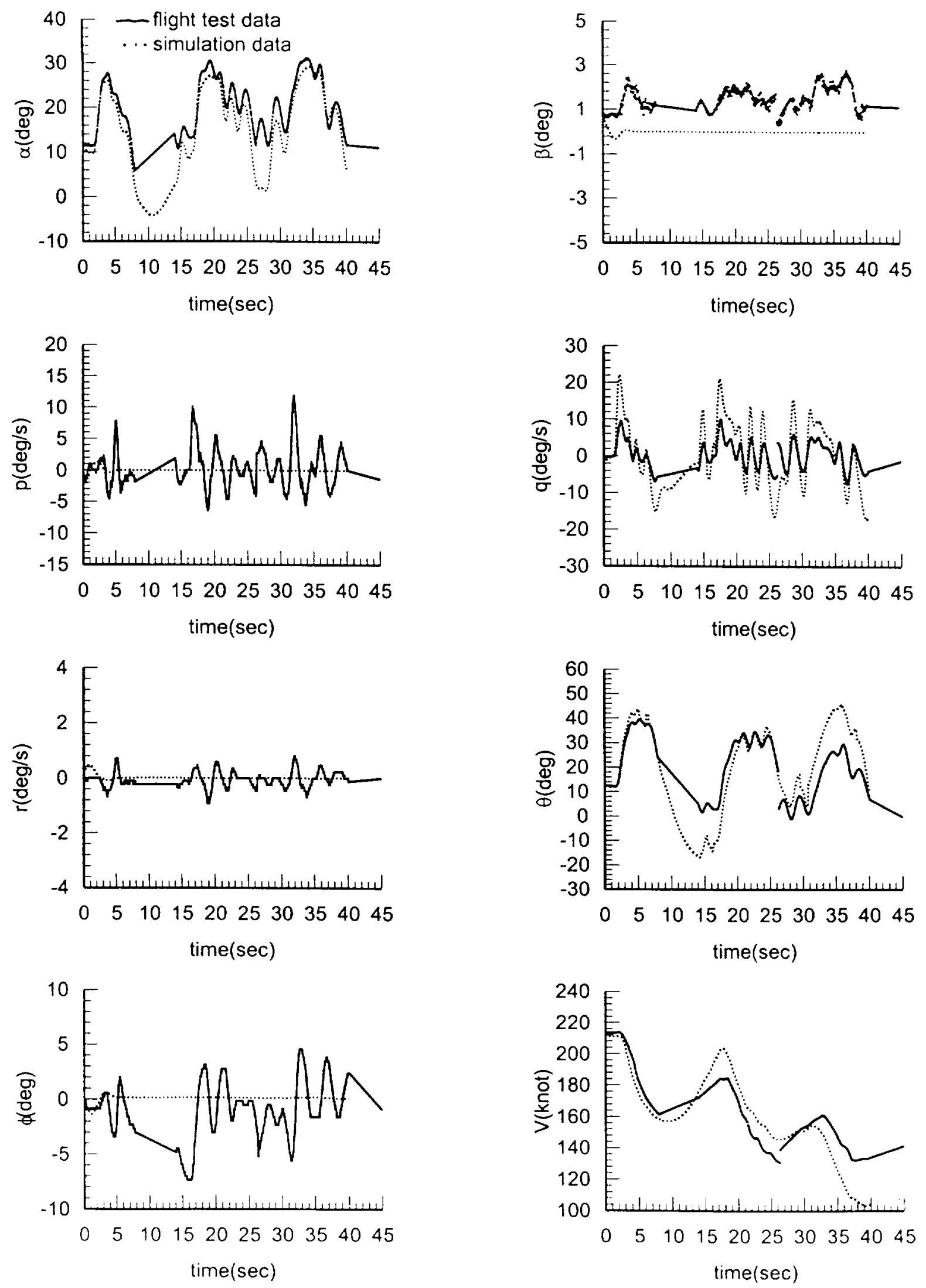

Figure 4 Comparison between flight test data and simulation data with quasi-steady aerodynamics at $M=0.4243, H=14931.03 \mathrm{ft}$ and $x \mathrm{cg}=0.45923 \bar{c}$ 

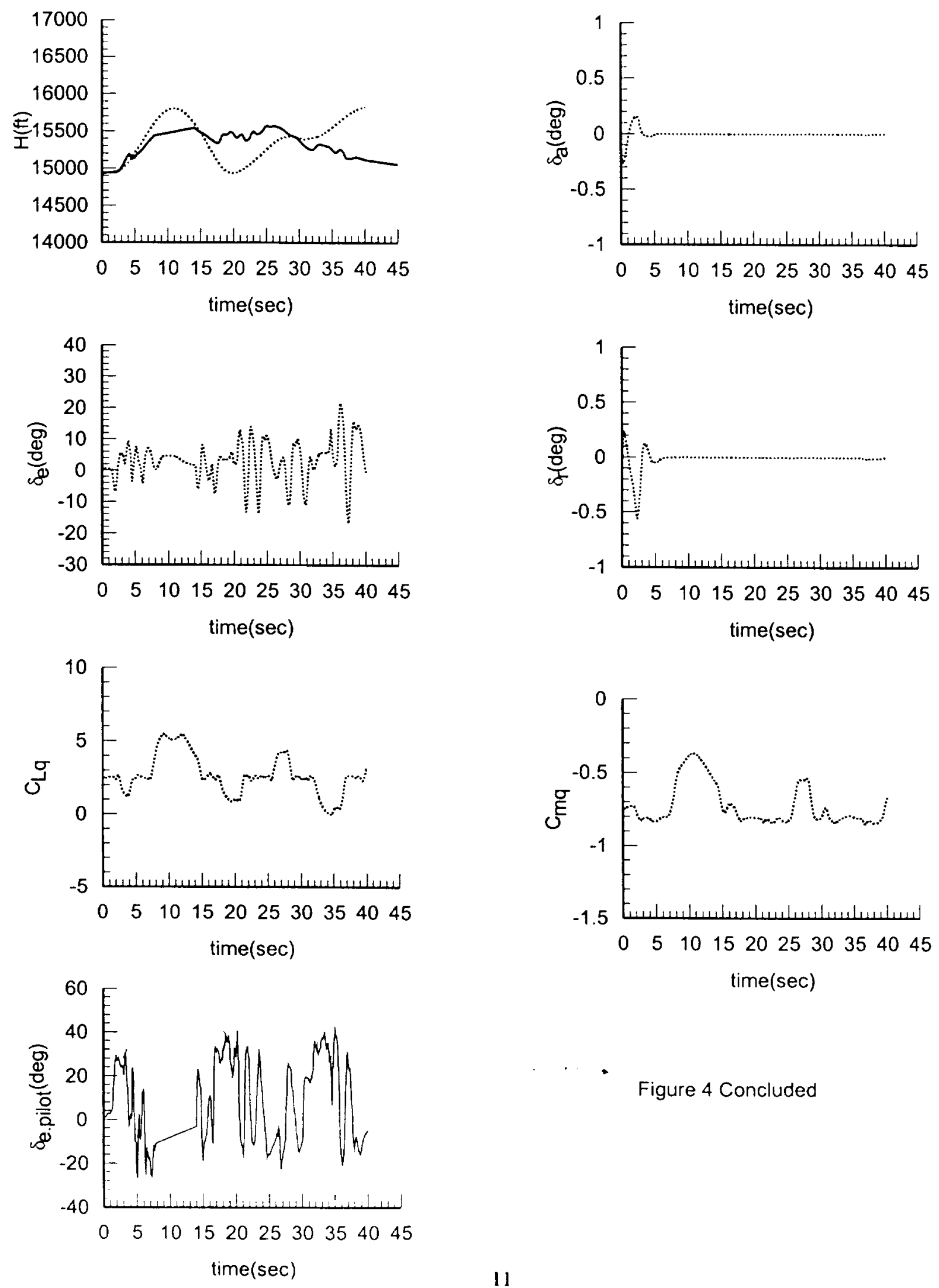

\section{Figure 4 Concluded}



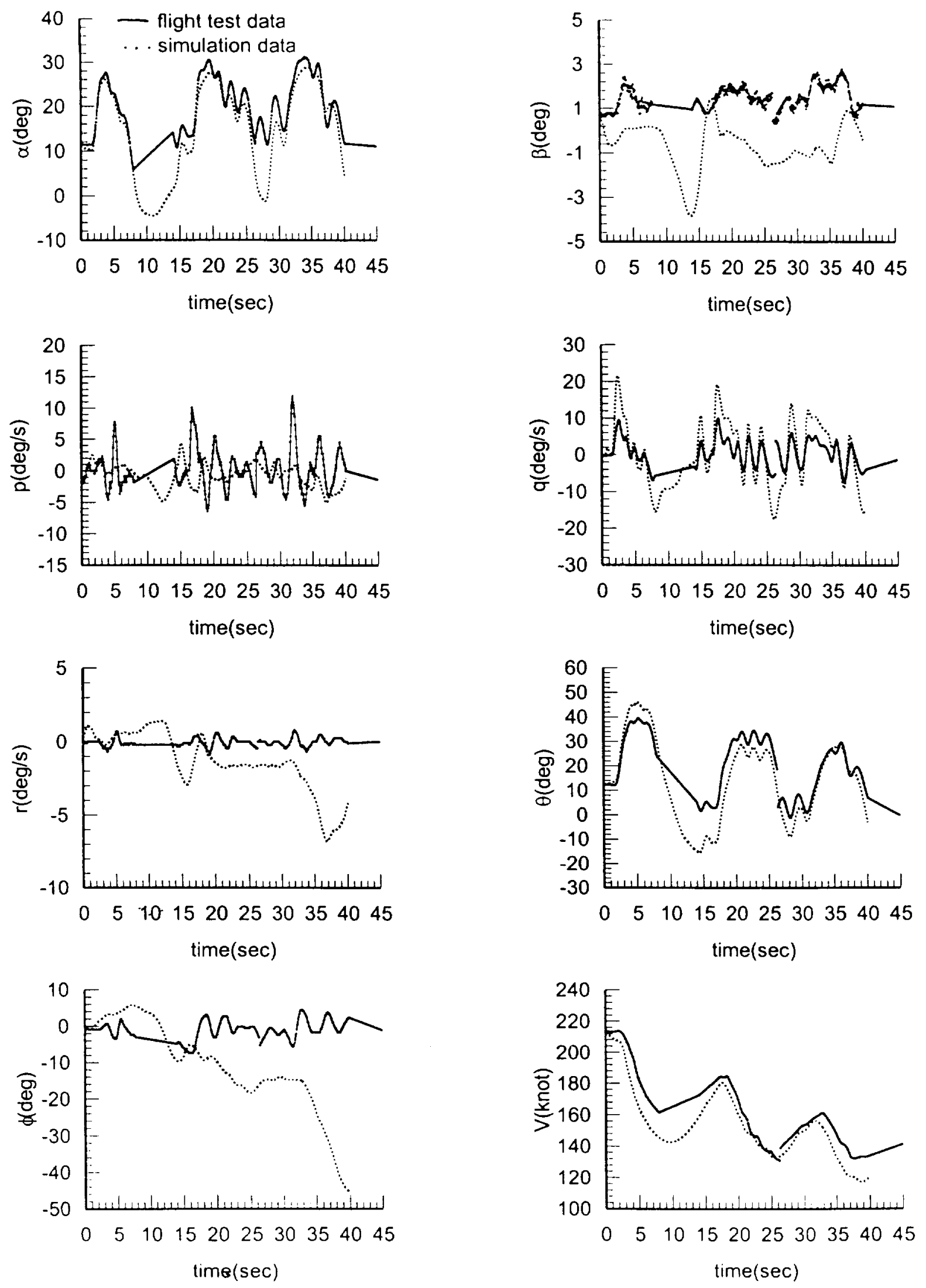

Figure 5 Comparison between flight test data and simulation data with unsteady aerodynamic effects at $M=0.4243, H=14931.03 \mathrm{ft}$ and $\mathrm{xcg}=0.45923 \overline{\mathrm{C}}$ 

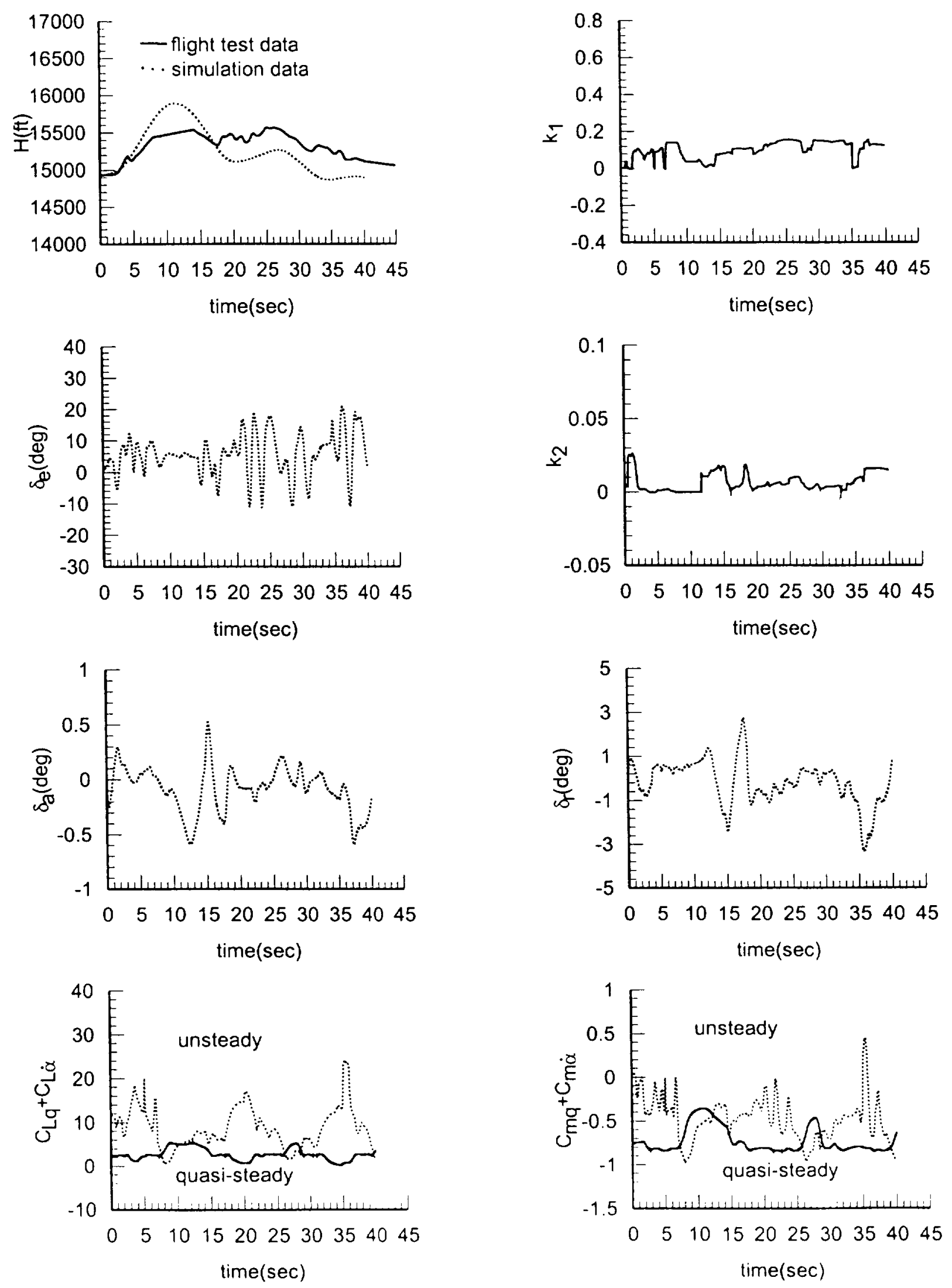

Figure 5 Concluded 

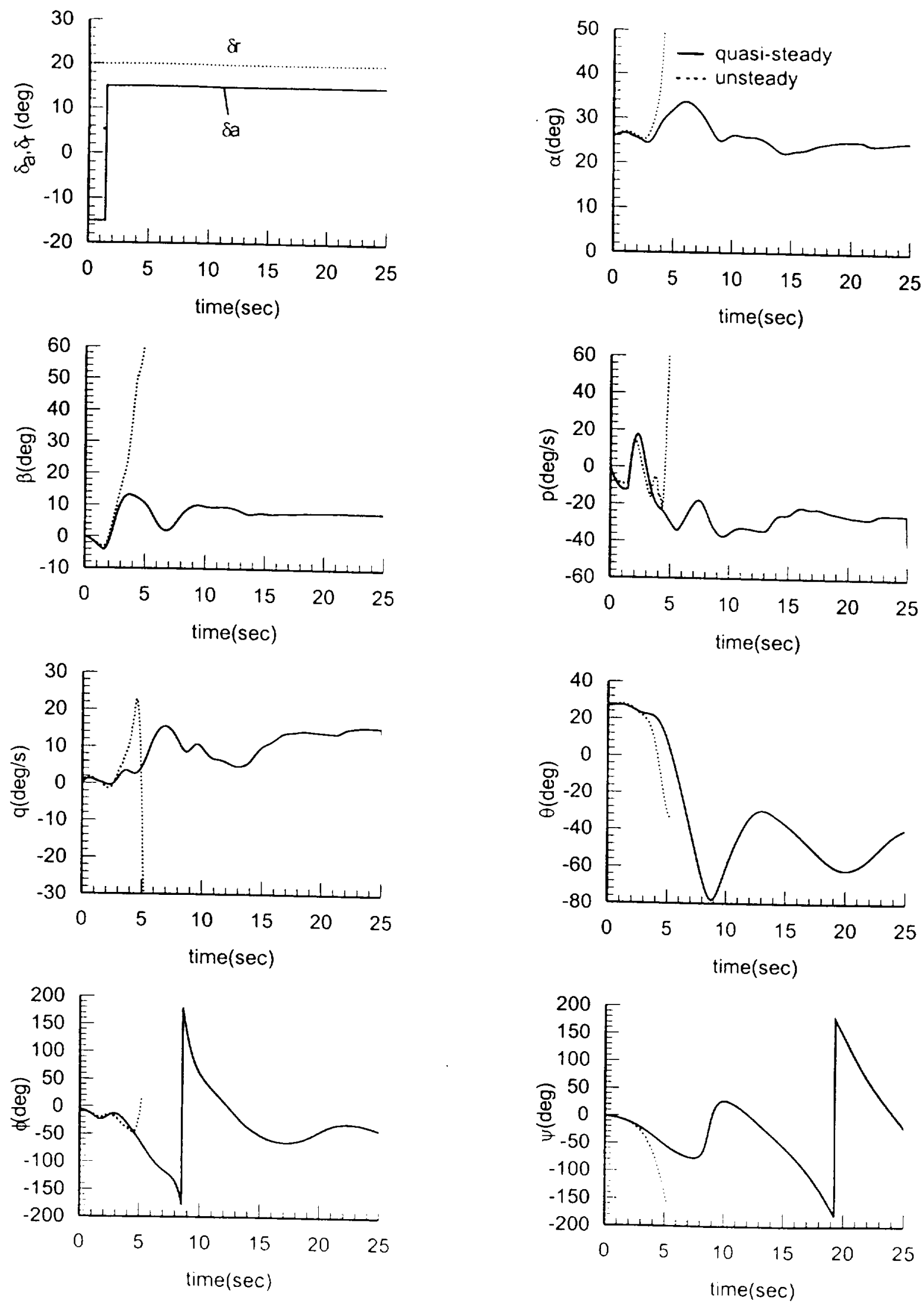

Figure 6 Comparison between the simulation results with and without unsteady aerodynamic effects at $M=0.1718, H=6000 \mathrm{ft}$ and $x \mathrm{cg}$ at $0.45923 \overline{\mathrm{C}}$ 

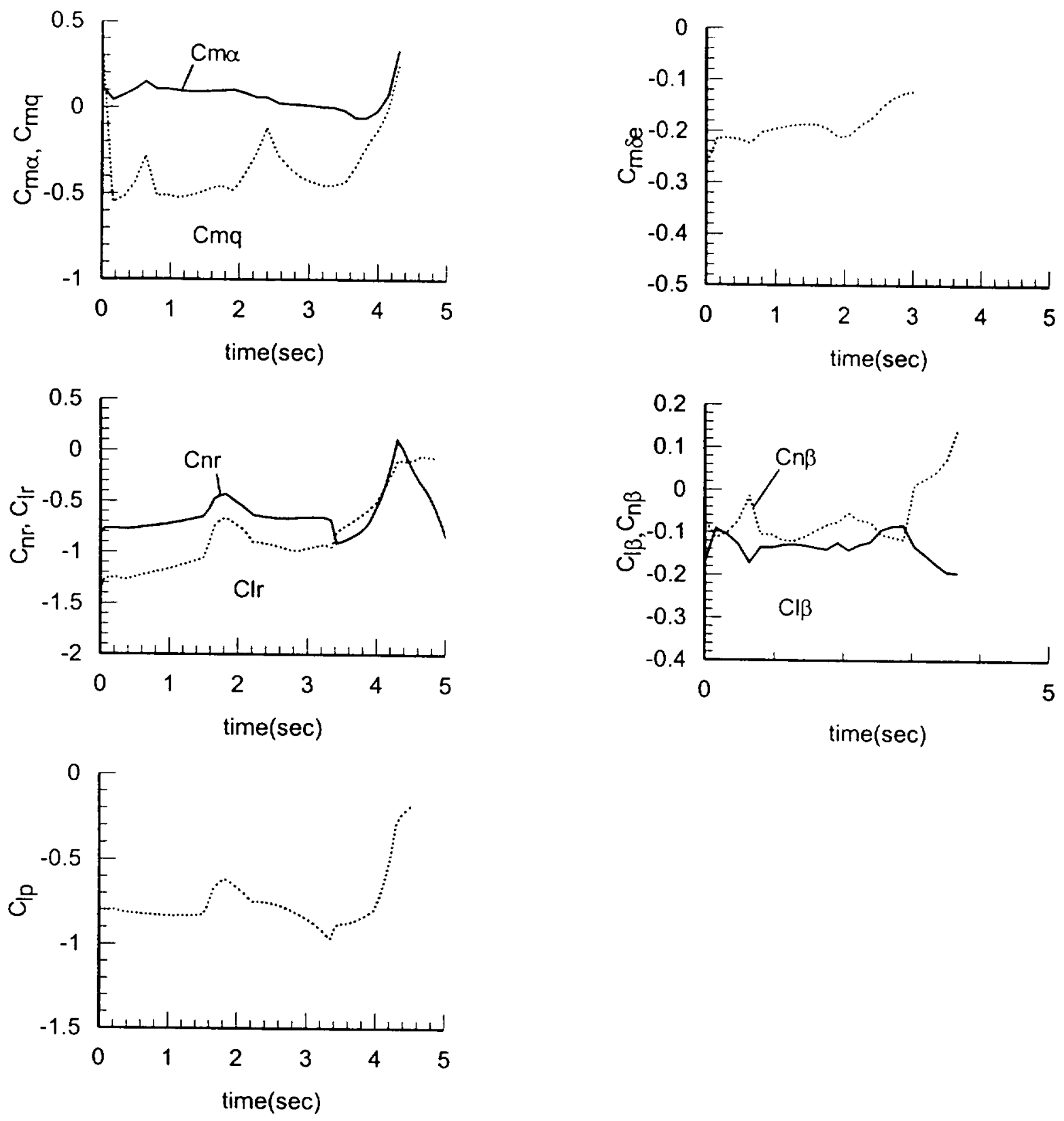

Figure 7 Stability derivatives of the unsteady configuration in flight conditions of Figure 6 